\title{
TOO LONG SHADOW BOUNDARIES
}

\author{
TUDOR ZAMFIRESCU
}

(Communicated by William J. Davis)

\begin{abstract}
It is shown that, in the sense of Baire categories, most $d$ dimensional convex bodies have infinitely long shadow boundaries if the light comes along one of many $(d-2)$-dimensional subspaces. This reveals (once again!) a striking contrast between the categorical and the measure-theoretical points of view.
\end{abstract}

This note only adds to several strange properties that most convex bodies enjoy (see the survey article [5]) a new one regarding their shadow boundaries. When we say "most" convex bodies, we always mean "all of them except those in a set of first Baire category". Roughly speaking, we discover that, while most convex bodies and their shadows are smooth, most of their shadow boundaries are terribly nonsmooth, in contrast to the title of [3]! To be more precise some definitions are needed.

Let $\mathscr{K}$ be the space of all convex bodies in $\mathbb{R}^{d}$. Equipped with Hausdorff's metric, $\mathscr{K}$ is a Baire space. The following result due to $\mathrm{V}$. Klee [2] and rediscovered by $\mathrm{P}$. Gruber [1] and others is fundamental:

PROPOSITION. Most convex bodies are smooth and strictly convex.

For a strengthening of the Proposition using the notion of porosity, see [6].

Let $\mathscr{G}_{k}$ be the grassmannian manifold of all $k$-dimensional linear subspaces of $\mathbb{R}^{d}$. For any $\alpha \in \mathscr{G}_{d-2}$, let $P_{\alpha} \in \mathscr{G}_{2}$ be orthogonal to $\alpha$. Now, for any $\alpha \in \mathscr{G}_{d-2}$ and $K \in \mathscr{K}$, let $p_{\alpha}: K \rightarrow P_{\alpha}$ be the orthogonal projection. If bd $K$ contains no linesegments parallel to $\alpha,\left.p_{\alpha}^{-1}\right|_{\mathrm{bd} p_{\alpha}(K)}$ is single-valued and continuous, where $\operatorname{bd} X$ means the relative boundary of $X$ (the boundary in aff $X$ ). The Jordan curve

$$
\Gamma(K, \alpha)=p_{\alpha}^{-1}\left(\mathrm{bd} p_{\alpha}(K)\right)
$$

is called the shadow boundary of $K$ along $\alpha$. Such a shadow boundary will be called singular if all its tangent lines (if any) are parallel to $\alpha$.

The preceding notions and following results may be formulated for $\mathscr{G}_{k}(1 \leq k \leq$ $d-2$ ) instead of $\mathscr{G}_{d-2}$. But on one hand there are obvious complications due to the fact that the new "shadow boundary" is no longer a curve, and, on the other, the interesting pathological behavior (see also Remark 3 at the end of this paper) is already well seen in case $k=d-2$.

THEOREM 1. Let $\alpha \in \mathscr{G}_{d-2}$. For most $K \in \mathscr{K}, \Gamma(K, \alpha)$ is singular.

ProOF. Since, by the Proposition, the space $\mathscr{K}^{+}$of all convex bodies without line-segments parallel to $\alpha$ on their boundaries is residual in $\mathscr{K}$, it makes sense to

Received by the editors November 23, 1986 and, in revised form, March 19, 1987.

1980 Mathematics Subject Classification (1985 Revision). Primary 52A20, 54E52. 
speak about $\Gamma(K, \alpha)$ and it suffices to show that, for most $K \in \mathscr{K}^{+}, \Gamma(K, \alpha)$ is singular.

At any point $x \in \mathbb{R}^{d}$, consider the cone

$$
K_{x}(\rho)=\left\{v \in \mathbb{R}^{d}: \exists w \in \alpha \text { with }\langle v-x, w\rangle \cdot\|v-x\|^{-1} \cdot\|w\|^{-1} \geq 1-\rho\right\}
$$

and let

$$
L_{x}(n)=\mathbb{R}^{d} \backslash \operatorname{int} K_{x}\left(n^{-1}\right) .
$$

If a shadow boundary $\Gamma(K, \alpha)$ is not singular then we can find a point $x$ in $\Gamma(K, \alpha)$ with $\Gamma(K, \alpha) \subset L_{x}(n)$ for some $n \in \mathbb{N}$. We have to show that the set $\mathscr{K}^{*}$ of all convex bodies $K \in \mathscr{K}^{+}$with nonsingular $\Gamma(K, \alpha)$ is of first category in $\mathscr{K}^{+}$. But $\mathscr{K}^{*} \subset \bigcup_{n=1}^{\infty} \mathscr{K}_{n}$, where $\mathscr{K}_{n}$ denotes the set of all $K \in \mathscr{K}^{+}$admitting an $x \in \Gamma(K, \alpha)$ with $\Gamma(K, \alpha) \subset L_{x}(n)$. $\mathscr{K}_{n}$ is closed. Indeed, let $K_{i} \in \mathscr{K}_{n}, K_{i} \rightarrow K$. Suppose the sequence of points $x_{i} \in K_{i}$ with $\Gamma(K, \alpha) \subset L_{x_{i}}(n)$ converges (otherwise choose a subsequence) to some point $x \in K$. Then $L_{x_{i}}(n)$ converges (in an obvious sense) to $L_{x}(n)$. Suppose there exists some $y \in \Gamma(K, \alpha) \backslash L_{x}(n)$. Let $V$ be a ball around $y$ disjoint from $L_{x}(n)$. Clearly, for some $i$ the supporting hyperplane of $K_{i}$ parallel to (and similarly situated as) that of $K$ at $y$ touches $K_{i}$ at a point of $V$, and $L_{x_{i}}(n)$ is still disjoint from $V$. Thus $\Gamma\left(K_{i}, \alpha\right) \not \subset L_{x_{i}}(n)$ and a contradiction is obtained. Hence $\Gamma(K, \alpha) \subset L_{x}(n)$ and $K \in \mathscr{K}_{n}$.

$\mathscr{K}^{+} \backslash \mathscr{K}_{n}$ is dense in $\mathscr{K}^{+}$. Indeed, let $\mathscr{O} \subset \mathscr{K}^{+}$be an open set. We find a polytope $P \in \mathscr{O}$. For every (maximal) line-segment $s$ in $\Gamma(P, \alpha)$, take a circular arc $A_{s}$ close to $p_{\alpha}(s)$ and with the same endpoints, but disjoint from int $p_{\alpha}(P)$, consider a line $l_{s} \subset P_{\alpha}$ orthogonal to $p_{\alpha}(s)$, take the 2-flat $\Pi_{s} \supset s$ parallel to $l_{s}$, and consider the points

$$
a_{1}(s), b_{1}(s), a_{2}(s), b_{2}(s), \ldots, a_{m_{s}}(s), b_{m_{s}}(s), a_{m_{s}+1}(s) \in \Pi_{s},
$$

whose orthogonal projections on $P_{\alpha}$ lie in this order on $A_{s}$, with the projections of $a_{1}(s)$ and $a_{m_{s}+1}(s)$ as the common endpoints of $A_{s}$ and $p_{\alpha}(s)$. Let $z \in \alpha$ and consider the points $b_{i}^{\prime}(s)=b_{i}(s)+z$ and the polytope

$$
Q=\operatorname{conv}\left(P \cup \bigcup_{s}\left\{a_{2}(s), a_{3}(s), \ldots, a_{m_{s}}(s), b_{1}^{\prime}(s), b_{2}^{\prime}(s), \ldots, b_{m_{s}}^{\prime}(s)\right\}\right) .
$$

If the $\operatorname{arcs} A_{s}$ are close enough to $p_{\alpha}(s)$ and $\|z\|$ is small enough, $Q \in \mathscr{O}$. If the pieces into which the projections of $b_{1}(s), a_{2}(s), b_{2}(s), \ldots, a_{m_{s}}(s), b_{m_{s}}(s)$ divide $A_{s}$ are small enough compared with $\|z\|$, then the slope (with respect to $P_{\alpha}$ ) of the line $l$ through $a_{i}(s)$ and $b_{i}^{\prime}(s)$ or through $b_{i}^{\prime}(s)$ and $a_{i+1}(s)$ becomes as large as desired, for example such that $l \subset K_{x}(1 / 2 n)$ for any $x \in l$. Since

$$
\Gamma(Q, \alpha)=\bigcup_{s} \bigcup_{i=1}^{m_{s}}\left(\operatorname{conv}\left\{a_{i}(s), b_{i}^{\prime}(s)\right\} \cup \operatorname{conv}\left\{b_{i}^{\prime}(s), a_{i+1}(s)\right\}\right),
$$

it follows that, for no $x \in \Gamma(Q, \alpha), \Gamma(Q, \alpha) \subset L_{x}(n)$, whence $Q \notin \mathscr{K}_{n}$.

Hence, being closed and having a dense complement, $\mathscr{K}_{n}$ is nowhere dense; therefore $\mathscr{K}^{*}$ is of first category.

THEOREM 2. For most $K \in \mathscr{K}$ and $\alpha \in \mathscr{G}_{d-2}, \Gamma(K, \alpha)$ is singular.

PROOF. Let $\mathscr{K}^{0}$ be the set of all strictly convex $K \in \mathscr{K}$. Since most convex bodies belong to $\mathscr{K}^{0}$, it suffices to prove the theorem for $\mathscr{K}^{0}$ instead of $\mathscr{K}$. 
Let $\mathscr{K}^{\prime}$ be the set of all convex bodies in $\mathscr{K}^{0}$ for which the set of all $\alpha \in \mathscr{G}_{d-2}$ providing a nonsingular shadow boundary is of second category. Let, for any $K \in$ $\mathscr{K}^{0}$

$$
A_{n}=\left\{\alpha \in \mathscr{G}_{d-2}: \exists x \in \Gamma(K, \alpha) \text { with } \Gamma(K, \alpha) \subset L_{x}(n)\right\} .
$$

Then

$$
\mathscr{K}^{\prime}=\left\{K \in \mathscr{K}^{0}: \bigcup_{n=1}^{\infty} A_{n} \text { is of second category }\right\} .
$$

Putting

$$
\mathscr{K}_{n}=\left\{K \in \mathscr{K}^{0}: A_{n} \text { is not nowhere dense }\right\}
$$

we may write

$$
\mathscr{K}^{\prime} \subset \bigcup_{n=1}^{\infty} \mathscr{K}_{n}
$$

and we only have to prove that $\mathscr{K}_{n}$ is of first category, for every $n \in \mathbb{N}$.

It is a routine matter to prove that $A_{n}$ is closed in $\mathscr{G}_{d-2}$. Thus, for $K \in \mathscr{K}_{n}$, there exists a nondegenerate ball in $A_{n}$. Let $\mathscr{K}_{n, m}$ be the set of all $K \in \mathscr{K}_{n}$ for which $A_{n}$ includes a ball of radius $m^{-1}$. Then

$$
\mathscr{K}_{n}=\bigcup_{m=1}^{\infty} \mathscr{K}_{n, m} .
$$

By the Proposition and Theorem 1, for a fixed $\alpha \in \mathscr{G}_{d-2}$ and for most convex bodies $K \in \mathscr{K}^{0}, \Gamma(K, \alpha)$ is singular.

Let now $\Sigma$ be a countable dense set in $\mathscr{G}_{d-2}$. Clearly,

$$
\mathscr{K}^{\prime \prime}=\left\{K \in \mathscr{K}^{0}: \forall \alpha \in \Sigma, \Gamma(K, \alpha) \text { is singular }\right\}
$$

is also residual in $\mathscr{K}^{0}$. Since $\mathscr{K}^{\prime \prime}$ and $\mathscr{K}_{n, m}$ are disjoint for any $n, m \in \mathbb{N}, \mathscr{K}_{n, m}$ is of first category and the proof is complete.

REMARK 1. Caution! Do not walk on a shadow boundary! If the body happens to be one of most of them, you will die before completing your trip. This must happen because every singular shadow boundary is nonrectifiable, which in turn follows from the fact that every nowhere differentiable function is not of bounded variation.

REMARK 2. A paper on the same topic by P. Gruber and H. Sorger using $\mathscr{G}_{k}$ with arbitrary $k$ is in preparation.

REMARK 3. There is a notable contrast between Theorem 2 and measuretheoretical results in [4]. P. Steenaerts [4] works in fact with analogously defined shadow boundaries $\Gamma(K, \alpha)$ in directions $\alpha \in \mathscr{G}_{1}$. He shows that, if $\gamma_{1}$ is the normalized Haar measure on $\mathscr{G}_{1}$ (so that $\gamma_{1}\left(\mathscr{G}_{1}\right)=1$ ) and $\lambda_{k}$ denotes the $k$-dimensional Hausdorff measure, then the average measure of shadow boundaries

$$
\Lambda(K)=\int_{\mathscr{S}_{1}} \lambda_{d-2}(\Gamma(K, \alpha)) d \gamma_{1}
$$

and the average measure of boundaries of projections

$$
\Xi(K)=\int_{\mathscr{S}_{1}} \lambda_{d-2}\left(\mathrm{bd} p_{\alpha}(K)\right) d \gamma_{1}
$$


verify $\Lambda(K) / \Xi(K) \leq d \pi^{-1} \omega_{d} \omega_{d-1}^{-1}$, from which one easily sees that $\Lambda(K)$ is finite. This in turn implies, as Steenaerts observes, that $\lambda_{d-2}(\Gamma(K, \alpha))$ is finite for almost every $\alpha \in \mathscr{G}_{1}$. In [3] the (more difficult) case $\alpha \in \mathscr{G}_{k}$ for arbitrary $k$ is considered, while the case $\alpha \in \mathscr{G}_{d-2}$ is discussed in $[4,9 \mathrm{~b}]$, with the same conclusion, namely that $\lambda_{1}(\Gamma(K, \alpha))<\infty$ for almost every $\alpha \in \mathscr{G}_{d-2}$.

Thus, for most $K \in \mathscr{K}, \Gamma(K, \alpha)$ is singular for most, but almost no $\alpha$ !

ACKNOWLEDGEMENT. Thanks are due to the referee for his valuable suggestions.

\section{REFERENCES}

1. P. Gruber, Die meisten konvexen Körper sind glatt, aber nicht zu glatt, Math. Ann. 229 (1977), 259-266.

2. V. Klee, Some new results on smoothness and rotundity in normed linear spaces, Math. Ann. 139 (1959), 51-63.

3. D. G. Larman and P. Mani, Almost all shadow boundaries are almost smooth, manuscript.

4. P. Steenaerts, Mittlere Schattengrenzenlänge konvexer Körper, Results in Math. 8 (1985), 5477.

5. T. Zamfirescu, Using Baire categories in geometry, Rend. Sem. Mat. Univ. Politec. Torino 43 (1985), 67-88.

6. - Nearly all convex bodies are smooth and strictly convex, Monatsh. Math. 103 (1987), 57-62.

Fachbereich Mathematik, Universität Dortmund, 4600 Dortmund, Federal REPUBLIC OF GERMANY 